This item was submitted to Loughborough's Research Repository by the author.

Items in Figshare are protected by copyright, with all rights reserved, unless otherwise indicated.

\title{
Application of an industrial robot in the sports domain: simulating the ground contact phase of running
}

PLEASE CITE THE PUBLISHED VERSION

http://dx.doi.org/10.1243/17543371JSET59

\section{PUBLISHER}

Professional Engineering Publishing (now Sage) and IMechE (@ The authors)

VERSION

VoR (Version of Record)

LICENCE

CC BY-NC-ND 4.0

\section{REPOSITORY RECORD}

Ronkainen, Jouni A., Rene El Kati, Paul R. Fleming, and Stephanie E. Forrester. 2019. "Application of an Industrial Robot in the Sports Domain: Simulating the Ground Contact Phase of Running". figshare. https://hdl.handle.net/2134/7690. 
This item was submitted to Loughborough's Institutional Repository (https://dspace.lboro.ac.uk/) by the author and is made available under the following Creative Commons Licence conditions.

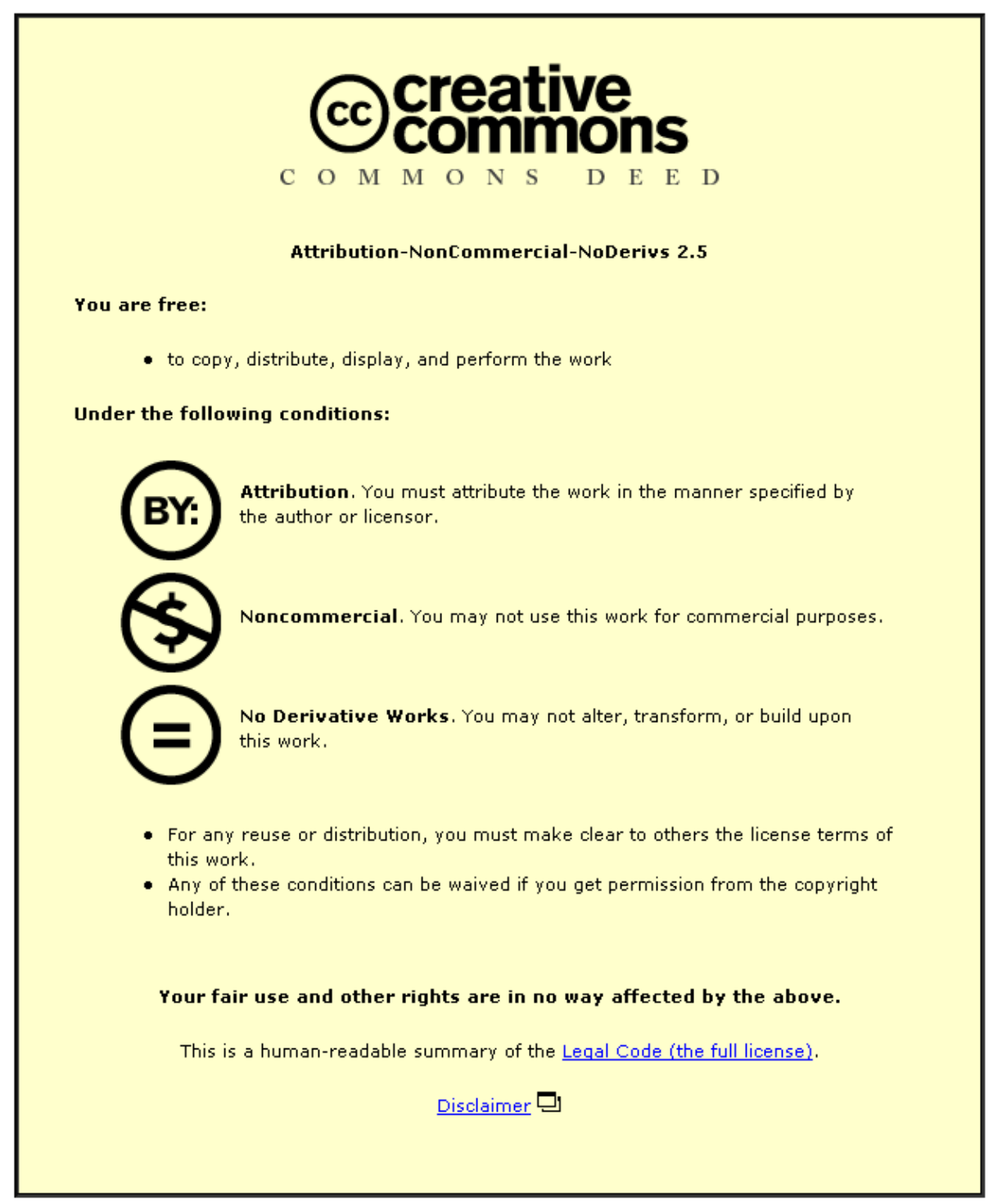

For the full text of this licence, please go to: http://creativecommons.org/licenses/by-nc-nd/2.5/ 


\title{
Application of an industrial robot in the sports domain: simulating the ground contact phase of running
}

\author{
J A Ronkainen ${ }^{1}$, R F El-Kati ${ }^{2}$, P R Fleming ${ }^{2}$, and S E Forrester ${ }^{1 *}$ \\ ${ }^{1}$ Wolfson School of Mechanical and Manufacturing Engineering, Loughborough University, Loughborough, UK \\ ${ }^{2}$ Department of Civil and Building Engineering, Loughborough University, Loughborough, UK
}

The manuscript was received on 21 September 2009 and was accepted after revision for publication on 6 May 2010.

DOI: $10.1243 / 17543371 J S E T 59$

\begin{abstract}
Mechanical devices currently used to test sports equipment are limited to one or two degrees of freedom and cannot replicate complete human movements. The purpose of this study was to investigate the capabilities of a six-degrees-of-freedom industrial robot (iRobot) to replicate the ground contact phase of human running. The objectives were as follows: to quantify the repeatability of the iRobot system; to assess the ability of the system to replicate heelstrike running and forefoot running.

High-speed video and force plate data were collected for a single-subject heelstrike running and forefoot running. The iRobot was programmed to replicate the two footstrikes and then to perform 500 cycles of each. System kinematics and ground contact forces were recorded every tenth cycle.

The kinematic repeatability of the iRobot was extremely good (less than $2 \mathrm{~mm}$ mean standard deviation in all marker trajectories). The peak vertical ground reaction forces showed systemic trends specific to the footstrike; heelstrike 3 per cent decrease and forefoot 19 per cent increase over the 500 cycles. iRobot replication of the footstrikes met with some success, particularly for the forefoot running.

The iRobot generated highly repeatable kinematics and demonstrated potential for applications within the footwear industry. A number of improvements to the system were identified which could further improve its ability to replicate human running.
\end{abstract}

Keywords: mechanical footwear tests, prosthetic foot, kinematics, ground reaction forces

\section{INTRODUCTION}

The sporting goods industry and research institutions commonly use a combination of human, mechanical, and virtual tests in the development of sports equipment. Human, or athlete, testing may provide the most realistic environment but numerous issues such as the sample size, variability in performance, number of trials, and data-processing times $[\mathbf{1}, \mathbf{2}]$ have meant that alternative methods are also heavily

*Corresponding author: Wolfson School of Mechanical and Manufacturing Engineering, Loughborough University, Loughborough, Leicestershire LE11 3TU, UK.

email: S.Forrester@lboro.ac.uk utilized. Virtual testing, such as finite element modelling, can be classed as a 'developing technology' with great potential as a cost- and time-effective means of equipment development. However, its current limitations include the geometric complexity of what can be modelled, the material properties, and the appropriate boundary conditions [3]. Consequently, mechanical testing remains an integral part in the development of sports equipment [4-8]. Mechanical devices are favoured because of their ease of use, controllability, and repeatability potentially over several thousand cycles. However, they are currently limited to only one or two linear or rotational degrees of freedom and thus lack biofidelity in their representation of human movements which are generally significantly more complex, e.g. running [9]. 


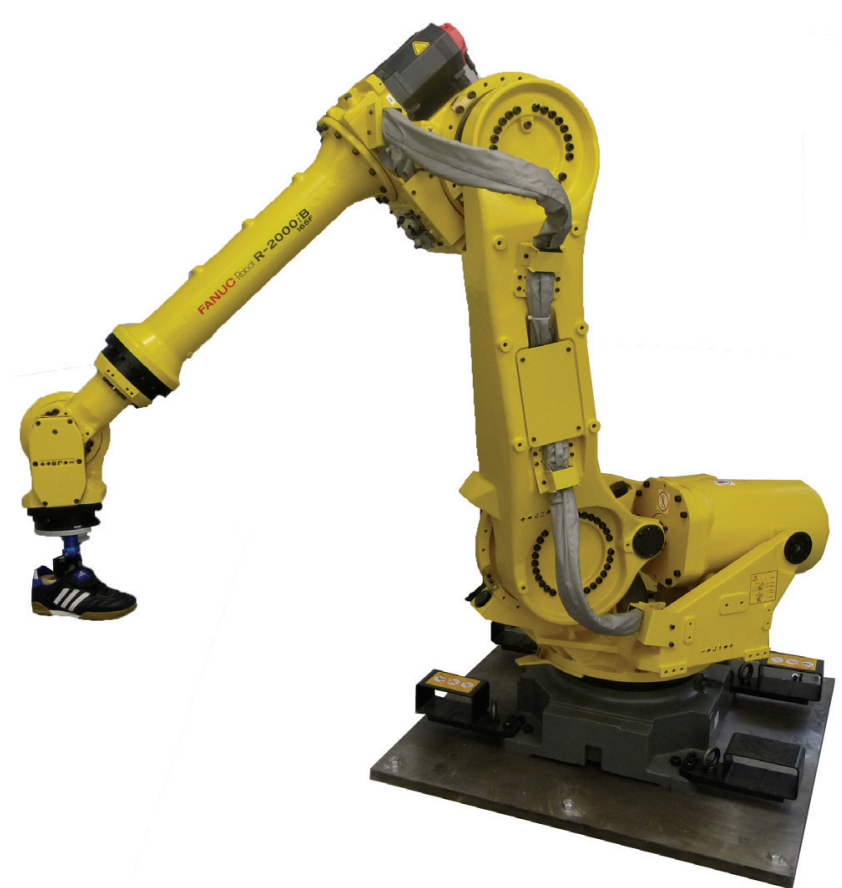

Fig. 1 The 6DOF Fanuc iRobot with shod prosthetic foot end effector attached

Despite the widespread use of six-degrees-offreedom (6DOF) mechanical devices across many industrial sectors such as automotive and electronics, very few attempts have been made to transfer this technology to sporting goods. Exceptions to this include the 6DOF Stewart-Gough platform 'automated footwear-testing system' device developed by Monckton and Chrystall [10] and Lewis et al. [11] and the Roboleg football kicker developed by Schempf [5]. In both cases, difficulties in the control systems of these devices led to early abandonment of the technologies for the given applications. The opportunities for a biofidelic mechanical testing device are such that, despite these earlier problems, continued investigation in this area is warranted. This research is centred on exploring the implementation of a 6DOF industrial robot (iRobot, Fanuc R-2000iB/165F (Fig. 1)) in the sporting domain. In contrast with the Stewart-Gough platform, the iRobot has a serial topology construction giving a larger working volume, a simplified approach to motion programming, and the ability to test a wider range of sports equipment. In contrast with the Roboleg, the iRobot is based on well-established and supported technology and is currently widely used for a variety of tasks across a range of industries. The successful implementation of such a robot into the sporting domain would potentially allow accurate replication of complex human movements.

A key stage in the development of footwear products is to ensure that they are fit for purpose, which encompasses a multitude of considerations such as comfort, aesthetics, durability, and functionality. At present, this is generally addressed through a combination of mechanical and human testing. The mechanical tests employ simple devices that are useful in providing the engineer with a measurement that can be used to benchmark shoes, e.g. single-axis impactors to measure heel-cushioning properties $[4,12,13]$ or machines to measure midsole bending stiffness [14]. However, these tests do not even attempt to replicate the dynamic loading conditions of human running. In order to test whole-shoe performance under realistic conditions, e.g. the durability to withstand typical loads during use, human trials are used which are time consuming, costly, and have low repeatability. Consequently, a mechanical test capable of replicating the dynamics of the human footstrike, and thus replacing many of the human trials, would have wide appeal and potentially a significant impact in this dynamic and competitive industry. The complexity of the ground contact phase of human running [9] indicates that this could only be achieved accurately using a 6DOF device.

This paper presents the first study in the public domain investigating the potential of a commercially available 6DOF industrial robot to test sports equipment. The iRobot system consisted of the kinematically controlled iRobot, a shod prosthetic right foot (Elite 2, Blatchford, UK) end effector, and twodimensional (2D) input kinematics. The aim was to determine whether it could repeatably and accurately replicate a human footstrike. It was hypothesized that the iRobot kinematics would be highly repeatable, to within specification $[\mathbf{1 5}]$, while the end-effector kinematics and ground contact forces would show lower repeatability, owing to the nonrigid nature of the component parts, but still better than typical intra-subject repeatability in human running trials. It was further hypothesized that the iRobot system would have only limited success in replicating human heelstrike running and forefoot running; in particular, the impact phase would be better replicated than the active (push-off) phase because of the limited flexion at the metatarsophalangeal joint (MPJ) of the prosthetic foot. The experimental design focused on recording human running trials for a single participant and using these data to programme the iRobot system. The iRobot then completed 500 cycles of the human running ground contact phase and the kinematics and ground contact forces measured for every tenth cycle. The results were also considered with respect to the feasibility of using the iRobot in a broader context of being able to simulate complex human motion to aid in the research and development of sports equipment. 


\section{METHODOLOGY}

The study was carried out in two phases. In the first, data were collected for human heelstrike running and forefoot running; in the second, the iRobot was programmed using the human data and then trials conducted involving multiple cycles of the two footstrikes.

\subsection{Athlete data collection}

One healthy male recreational athlete (age, 26 years; height, $1.75 \mathrm{~m}$; weight, $67 \mathrm{kgf}$; shoe size, UK8) provided written informed consent and the study was conducted in accordance with the approval given by Loughborough University Ethical Advisory Committee.

The athlete was asked to perform heelstrike running and forefoot running trials at a consistent self-selected running speed $(3.6 \pm 0.2 \mathrm{~m} / \mathrm{s})$. A force platform (Kistler 9281CA; $1000 \mathrm{~Hz}$ ) was positioned in the centre of the laboratory and a successful trial defined as when the subject's right foot made contact with the centre of the force platform. Five successful trials were collected for each running technique. The 2D lower-leg kinematics corresponding to the ground contact phase on the force platform were obtained using a single high-speed video camera (Photron FASTCAM SA1.1; $500 \mathrm{~Hz}$ and $\frac{1}{2000} \mathrm{~s}$ shutter speed) synchronized with the force platform. The video images were manually digitized (Image-Pro Plus) to give $2 \mathrm{D}$ coordinates of the toe, the base of the fifth MPJ, and the heel from immediately prior to impact until post toe-off.

\section{2 iRobot system}

From preliminary tests, a top-of-the-range athletic prosthetic foot (Elite 2, Blatchford (Fig. 2)) was preferred to a rigid foot last as the end effector as it provided more realistic human motion. A qualitative comparison using high-speed video footage on the performance of both end effectors relative to the human trials indicated that the prosthetic foot provided local compression at heelstrike (through the carbon fibre spring mechanism) and flexion at the MPJ when loading was on the forefoot region (through the carbon fibre blades that ran the length of the foot). The end-effector kinematics were programmed using a point-to-point protocol based on the position and velocity of the heel marker and the angle of the shoe sole in a global reference frame as obtained from representative human running trials. This provided 34 discrete points for heelstrike running and 29 for forefoot running starting immediately prior to touchdown and ending immediately post toe-off. It was also necessary to specify a level of smoothing, and this was set to give a smooth motion of

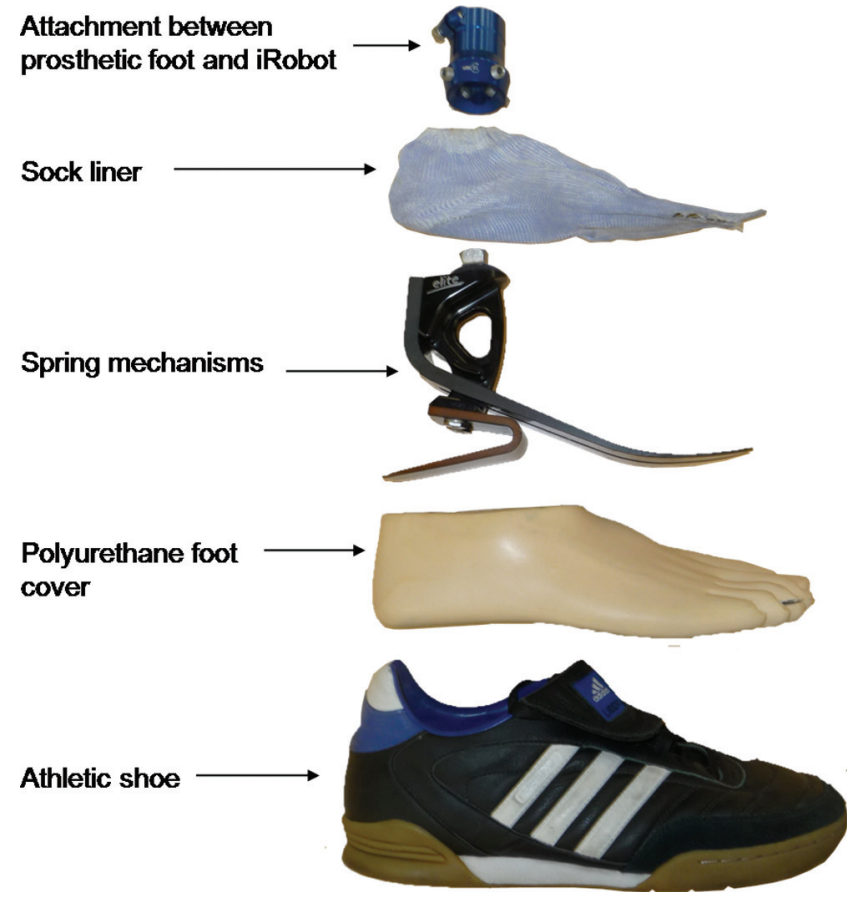

Fig. 2 Component parts of the shod prosthetic foot end effector

the end effector throughout footstrike. The smoothing is controlled within the iRobot control software where the user defines a level of between 0 per cent and 100 per cent. 0 per cent forces the iRobot through every programmed point, where it will stop momentarily at each point, leading to a very jerky motion with high accelerations and decelerations. 100 per cent gives a heavily smoothed trajectory in which the iRobot bypasses the intermediate programmed points in moving from the start to the end point; however, there is no slowing at each point and no jerk. The vertical position of the whole trajectory was lowered on to the force platform until the peak vertical ground reaction force (vGRF) matched that recorded in the human trial. The start and end positions for each of the two footstrikes were with the whole foot airborne.

A force platform, similar to that used for athlete testing (Kistler 9281CA; $1000 \mathrm{~Hz}$ ), was installed within the iRobot enclosure. The platform was mounted on four nitrogen springs, one at each corner, set to depress if the loading exceeded $5 \mathrm{kN}$. This was deemed necessary owing to the lack of force control on the iRobot; if the maximum load capacity of the platform was reached, the springs would dissipate the load and prevent damage to the force platform.

\section{3 iRobot data collection}

A Codamotion analysis system (CX1, Charnwood Dynamics; $400 \mathrm{~Hz}$ ) measured the kinematics of the 


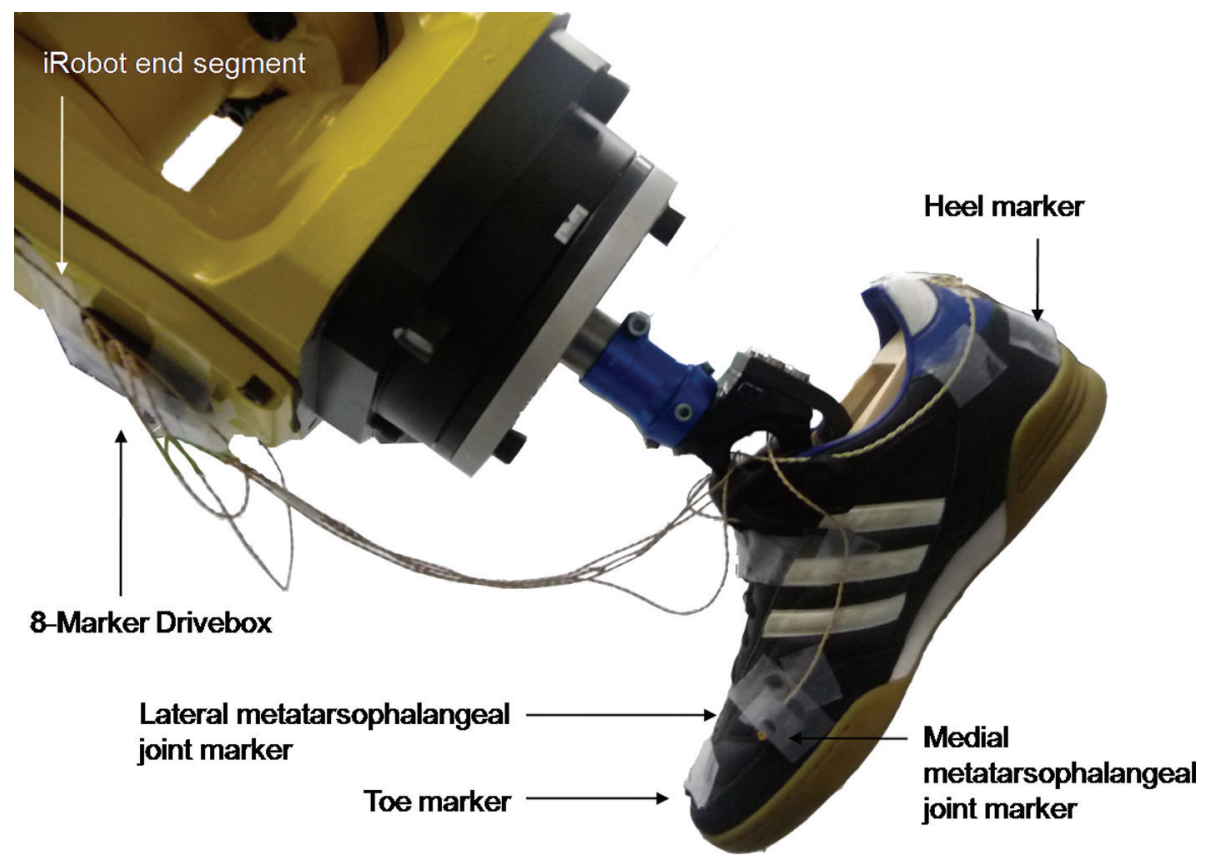

Fig. 3 Codamotion marker placements for the iRobot testing

iRobot and the end effector using three capture heads positioned in the iRobot enclosure. A total of seven markers were used (Fig. 3) with three on the terminal iRobot segment (lateral, medial, and lower) and four on the running shoe (toe, medial, and lateral MPJ markers, and heel) where the footwear was the same as used in the athlete testing. The Kistler force platform was connected to an amplifier control unit (Kistler 5233A) and thereafter to an analogue-to-digital converter within the Codamotion hardware. The Codamotion synchronous output triggered the force platform to start recording at the same time as the Codamotion system, thus providing synchronous kinematic and ground reaction force data. The iRobot was set to conduct 500 continuous cycles of each footstrike, with each cycle taking approximately $5 \mathrm{~s}$. The total of 1000 cycles took approximately $3 \mathrm{~h}$ to complete which included the time required to switch between the two footstrike patterns. Kinematic and force platform data were collected every tenth cycle and thus, for both gait conditions, 50 data captures were achieved. Each capture lasted $2 \mathrm{~s}$ and all data were exported to MATLAB for processing. The force platform was reset between captures, removing the potential for drift associated with long capture durations.

\subsection{Data processing}

The ground contact phase for each footstrike was identified on the basis of a force threshold and only data from this phase were processed further. The trajectories for each marker were averaged over the 50 data captures and the mean standard deviation (MSD) evaluated as a measure of trajectory repeatability. The peak vGRF was also used as a repeatability test. Replication of the footstrikes was based on the vertical and horizontal (anterior-posterior) force profiles, ground contact time, centre-of-pressure (COP) location, and MPJ angle estimated from the heel, lateral MPJ, and toe marker positions. A similar process was conducted for the athlete testing data.

\subsection{Safety considerations}

Safety was an important concern for the iRobot since the end segment was capable of withstanding a maximum payload of $165 \mathrm{kgf}$, linear velocities up to $2 \mathrm{~m} / \mathrm{s}$, and a maximum reach of $2.65 \mathrm{~m}$. Therefore stringent safety protocols were developed which included containing the iRobot within a Plexiglas cage lined with five light curtains which instantaneously tripped the iRobot if their field of view was impeded.

\section{RESULTS}

\subsection{Repeatability of the iRobot system}

The iRobot end segment marker trajectories were highly repeatable throughout the 500 cycles for both footstrikes (Table 1). The MSDs for all iRobot trajectories were less than $2 \mathrm{~mm}$, corresponding to less than 1 per cent of total trajectory length. The repeatability for the heelstrike running action was slightly 
better than for the forefoot action with the MSD smaller for most marker trajectories. Only for the lower and lateral iRobot markers in the $x$ direction (medial-lateral) was the MSD higher; however, movement in this plane was minimal.

The end-effector marker trajectories were also highly repeatable, although they had slightly higher MSDs (less than $4 \mathrm{~mm}$ ) than those on the iRobot (Table 1). Similarly to the iRobot markers, the repeatability was slightly better for the heelstrike running action than for the forefoot action. An interesting observation was that the MSDs were the same for the toe marker and the medial MPJ marker, and this was the case for all trajectories and both heelstrike running and forefoot running.

The peak vGRF showed systematic trends across the 500 cycles which were footstrike specific (Fig. 4). For the heelstrike, it decreased by 3 per cent while, for the forefoot, it increased by 19 per cent. Furthermore, for the forefoot running peak, the vGRF seemed to fall on one of two distinct curves. These results were further reflected in the coefficient-of-variation (CV) data for the force platform parameters (Table 2) which

Table 1 The MSDs of the marker trajectories throughout the ground contact phase of running, which were obtained by averaging the 50 trials representing every tenth ground contact over 500 footstrike cycles ( $x$, medial-lateral; $y$, anterior-posterior; $z$, vertical)

\begin{tabular}{|c|c|c|c|c|c|c|}
\hline & \multicolumn{6}{|c|}{$\begin{array}{l}\text { MSD (mm) for the following } \\
\text { footstrikes and directions }\end{array}$} \\
\hline & \multicolumn{3}{|c|}{ Forefoot } & \multicolumn{3}{|c|}{ Heelstrike } \\
\hline & $x$ & $y$ & $z$ & $x$ & $y$ & $z$ \\
\hline Lower iRobot & 0.180 & 1.487 & 0.710 & 0.323 & 0.392 & 0.228 \\
\hline Medial iRobot & 0.123 & 1.524 & 0.727 & 0.063 & 0.420 & 0.234 \\
\hline Lateral iRobot & 0.157 & 1.565 & 0.728 & 0.240 & 0.440 & 0.223 \\
\hline Toe & 0.343 & 3.677 & 1.695 & 0.267 & 1.024 & 0.220 \\
\hline Medial MPJ & 0.343 & 3.677 & 1.695 & 0.267 & 1.024 & 0.220 \\
\hline Lateral MPJ & 0.999 & 3.751 & 1.007 & 0.279 & 1.234 & 0.266 \\
\hline Heel & 0.252 & 2.225 & 3.775 & 0.085 & 0.217 & 0.254 \\
\hline
\end{tabular}

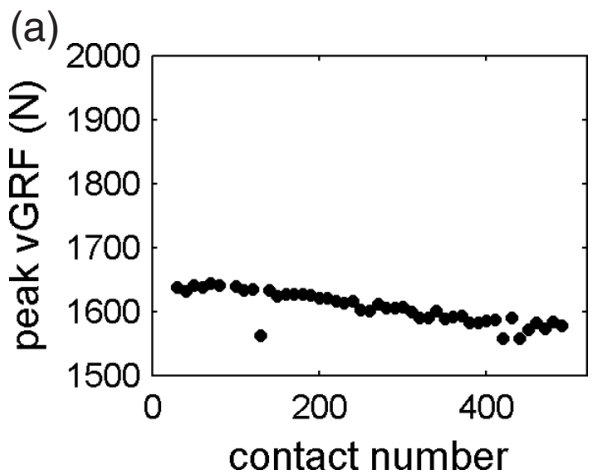

were lower for heelstrike running (not more than 1.6 per cent) than for forefoot running (not more than 5.1 per cent). Thus, the heelstrike condition appeared to be the more repeatable at the foot-ground interface.

\subsection{Replication of the foot-ground contact phase of human running}

Qualitatively there appeared to be good agreement between the human and iRobot running apart from at the MPJ, where the iRobot end effector did not flex as much as the human foot during the second half of stance (Fig. 5). Specifically, the image sequences indicate that the prosthetic foot kinematics were similar to those of the human foot with the main discrepancy being towards toe-off where the prosthetic foot developed less flexion at the MPJ. Quantitatively, correspondence between the human running and iRobot running gave mixed results and overall was better for forefoot running than for heelstrike running (Table 2 and Fig. 6). In particular, the forefoot running action had good correspondence in the vGRF whereas the heelstrike running action in the iRobot generated only a single peak in the vGRF, which occurred later in the stance phase than either the impact or active peaks in the human running. The horizontal ground reaction forces (hGRFs) were negative (braking) throughout the stance for both iRobot footstrikes, in contrast with the human trials, which illustrated a braking phase followed by a propulsive phase. The iRobot had ground contact times that were twice to three times longer than the human running, and vertical loading rates were up to an order of magnitude too low.

The iRobot smoothing had a marked effect on the foot kinematics, as illustrated in the heel marker trajectories for the human and iRobot (Fig. 7). In the human trials for both heelstrike running and forefoot running, there was a sharp change in the direction early in the heel marker trajectory; however, this was absent in the iRobot trajectory, which was smooth throughout ground contact. (b)

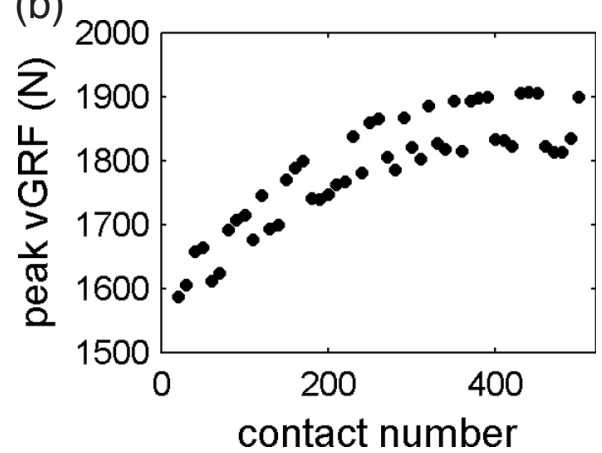

Fig. 4 Peak vGRFs for the iRobot tests: (a) heelstrike; (b) forefoot 
Table 2 Values of the mean, standard deviation (SD), and CV for the principal force platform parameters in the human and iRobot trials

\begin{tabular}{|c|c|c|c|c|c|c|}
\hline & \multicolumn{3}{|c|}{ Human } & \multicolumn{3}{|c|}{ iRobot } \\
\hline & Mean & SD & $\mathrm{CV}$ & Mean & SD & $\mathrm{CV}$ \\
\hline \multicolumn{7}{|l|}{ Heelstrike } \\
\hline Ground contact time (ms) & 216 & 10 & 4.6 & 612 & 5 & 0.8 \\
\hline Peak vGRF (BW) & 2.57 & 0.2 & 7.8 & 2.44 & 0.04 & 1.6 \\
\hline Time of peak vGRF (ms) & 57.7 & 26.3 & 46 & 472 & 3 & 0.6 \\
\hline Time of peak vGRF (\% contact time) & 26.6 & 11.5 & 43 & 77.0 & 0.2 & 0.3 \\
\hline Peak vertical loading rate (BW/s) & 131.7 & 27.2 & 20.7 & 11.4 & 0.1 & 0.9 \\
\hline \multicolumn{7}{|l|}{ Forefoot } \\
\hline Ground contact time (ms) & 164 & 3 & 1.8 & 331 & 9 & 2.7 \\
\hline Peak vGRF (BW) & 2.49 & 0.09 & 3.6 & 2.72 & 0.13 & 4.8 \\
\hline Time of peak vGRF (ms) & 84 & 5 & 6.0 & 159 & 7 & 4.4 \\
\hline Time of peak vGRF (\% contact time) & 51.5 & 2.5 & 4.9 & 48.0 & 1.1 & 2.3 \\
\hline Peak vertical loading rate (BW/s) & 84.3 & 3.8 & 4.5 & 23.7 & 1.2 & 5.1 \\
\hline
\end{tabular}

BW, body weight.

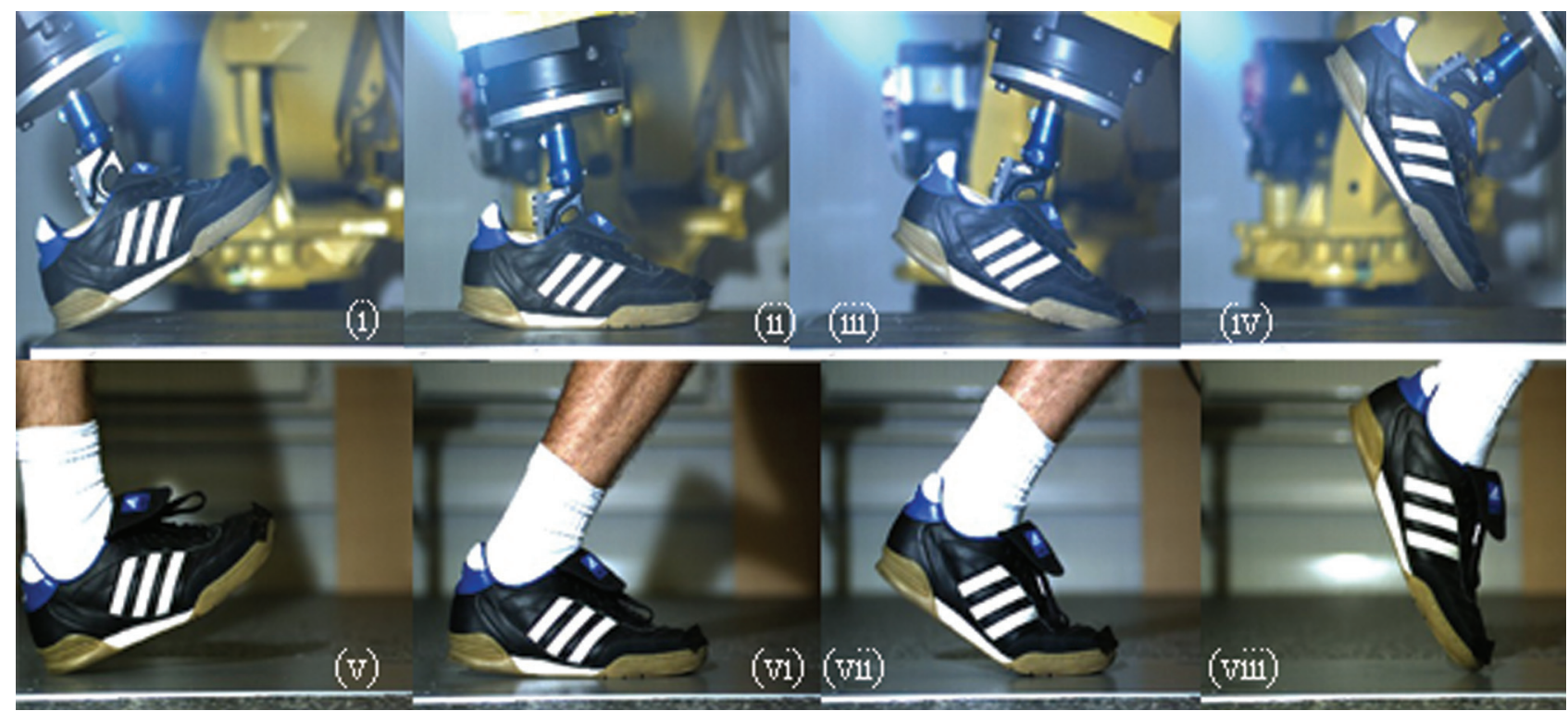

Fig. 5 Series of images from the iRobot running and human heelstrike running. The images show the iRobot end-effector kinematics ((i) heel contact; (ii) 35 per cent of total impact time; (iii) 66 per cent of total impact time; (iv) toe-off) and the human kinematics ((v) heel contact; (vi) 36 per cent of total impact time; (vii) 53 per cent of total impact time; (viii) toe-off)

\section{DISCUSSION}

The iRobot end segment and the end-effector kinematics were both highly repeatable over 500 footstrike cycles of heelstrike running and forefoot running. The iRobot kinematics were to within the accuracy of the Codamotion kinematic measurement system and supported the manufacturer's claims of repeatability to within $\pm 0.2 \mathrm{~mm}[\mathbf{1 5}]$. The shod prosthetic foot kinematics showed slightly higher variability, the magnitude of which was dependent on the marker position and footstrike technique. This increased variability is likely to have resulted from a number of contributing factors including the following: the non-rigidity of the component parts of the end effector (Fig. 2); the relative movements of these component parts; the movement of the force platform; the movements of the markers on the shoe; the wear of the shoe and/or foot parts. Notably, the variability was greater for forefoot running, most probably because the prosthetic foot was not designed for this type of footstrike (bypassing 

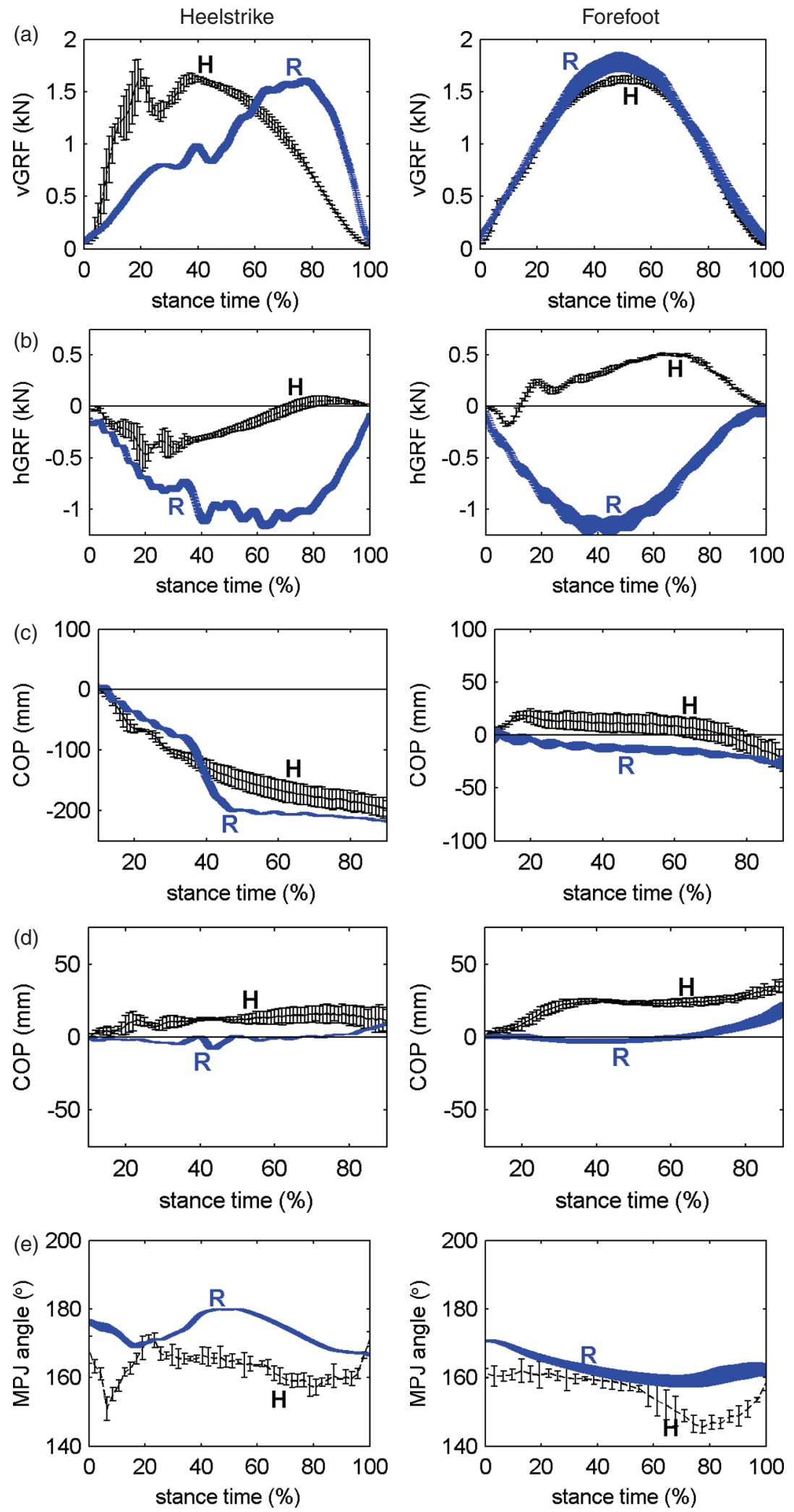

Fig. 6 Comparison between the human (curves H) running trials and the iRobot (curves R) running trials. The graphs show the mean \pm SD throughout the ground contact phase for (a) the vGRF, (b) the hGRF, (c) COP $y$ location, (d) COP $x$ location, and (e) the MPJ angle 

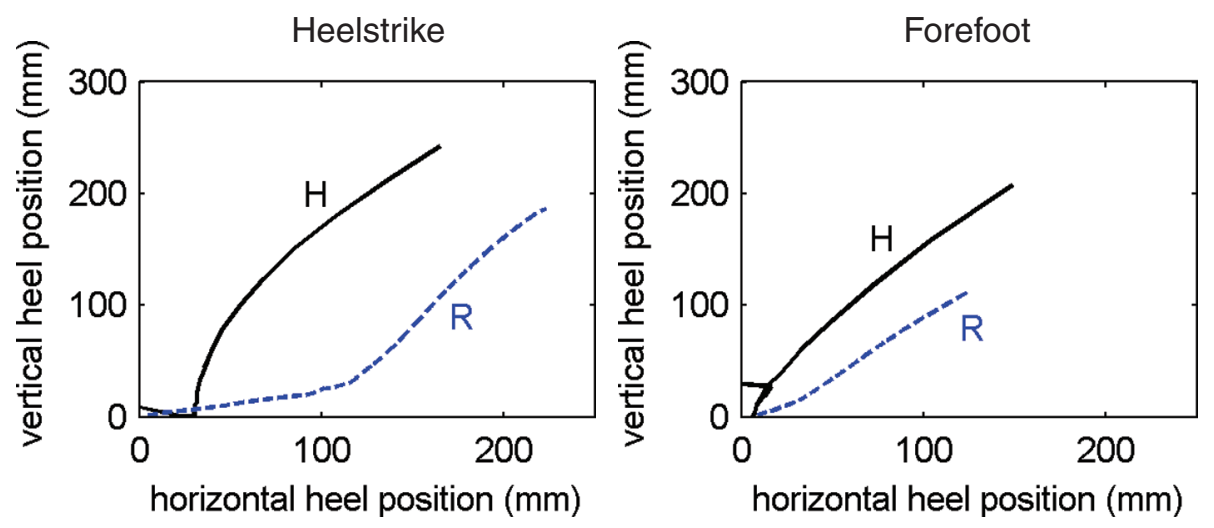

Fig. 7 Comparison between the heel marker trajectories during ground contact in the human (curves $\mathrm{H}$ ) running and iRobot (curves $\mathrm{R}$ ) running trials

the heel spring mechanism (Fig. 2)) compounded by the steeper angle of attack on to the force platform. Indeed, the forefoot trials led to marked degradation of the prosthetic foot during the 500 cycles with the toe end of the spring mechanism almost puncturing the sockliner and polyurethane foot cover.

Similarly, the repeatability of the peak vGRF was good for heelstrike running but much poorer for forefoot running. For the latter the peaks seemed to fall on two distinct curves, both displaying a trend of increasing force as cycling progressed. Again, this may be related to the prosthetic foot design, which possibly resulted in some relative slippage between the shoe and foot components, producing two distinct impact conditions. For heelstrike running, the observed trends are likely to be a result of wear in the shoe (small decrease in the peak force) but, for forefoot running, they are more likely to be dominated by changes in the impact conditions due to the relative movement described above and to actual damage of the prosthetic device.

It is of interest to compare the magnitudes of the variability in the iRobot system parameters with that typically observed in human running. This has relevance in ensuring that the iRobot system has lower variability than human movements and thus represents a reliable test device; however, it may also be of interest in assessing the future potential of the iRobot to replicate not only the human movement but also the inherent variability in that movement [16]. An initial comparison can be made on the basis of the variability in the human movement data captured for this study, and this supports the fact that the iRobot has the lower variability (CV data in Table 2 and Fig. 6). However, since only five human trials for each footstrike were collected and with minimal control over the running velocity, then a further comparison using literature data is necessary. Little information exists for variability in human running; however, significant data exist for walking.
CVs over multiple trials for the vGRF are generally reported to be in the range 4-14 per cent and for the hGRF in the range 10-20 per cent [17-19]. These values are significantly higher than the CVs for the vertical and horizontal forces in the iRobot trials, particularly for the heelstrike tests. Again, this supports the high repeatability of the iRobot system, to a greater level than is typically seen in actual human movement.

The human footstrikes were replicated with limited success. In contrast with the repeatability results, the system replicated the ground contact phase of forefoot running better than that of heelstrike running. This was particularly true for the vGRF, including the timing of the peak force during stance. The good agreement is likely to result from the relatively simple nature of the ground contact phase of forefoot running compared with that of heelstrike running, where the latter involves a transfer in load from landing on the heel to pushing off on the forefoot. Hence, although the end effector was not designed for forefoot running and indeed was damaged in the process of these trials, it performed well in replicating the kinematics and contact forces of the interaction. The reasons why heelstrike running is less well replicated are likely to be related to the complexity of the movement (including a significant three-dimensional (3D) component which was not represented here) combined with an end effector designed to provide stable support for a human walking rather than to replicate actual foot function. During the ground contact phase of running, in addition to movement in the main direction of travel (sagittal plane), the foot also pronates (principally a frontal plane movement $[\mathbf{2 0}]$ ), a mechanism thought to be important to reduce the impact loading experienced by the human [21]. For steady heelstrike running at $3.8 \mathrm{~m} / \mathrm{s}$, the rearfoot angle measured in the frontal plane and assumed to represent pronation changed by $15^{\circ}$ over the first 
$40 \mathrm{~ms}$ of impact [22]. In terms of ground reaction forces, for steady heelstrike running at $4.5 \mathrm{~m} / \mathrm{s}$, the peak vertical and peak-to-peak anterior-posterior and medial-lateral ground reaction forces have been reported at approximately $3 \mathrm{BW}, 1 \mathrm{BW}$, and $0.3 \mathrm{BW}$ respectively [23]. The more complex foot trajectory may have caused the smoothing used in the programming of the iRobot to have had a greater effect for heelstrike running than for forefoot running. The level of smoothing was selected to avoid unrealistic jerks in the end-effector movement but at the expense of a foot trajectory that differed somewhat from that input from the human trials. Typical applications of the iRobot will require the start point and end point of the end-effector movement to be very accurately defined; however, and in contrast with the current study, the exact trajectory is less important and this is reflected in the control system. In future studies, it would be relevant to investigate the effect of different levels of smoothing on both position and jerk profiles, aimed at establishing the level of smoothing which combines a satisfactory low level of jerk and the least amount of positional smoothing. Since the number and location of points used in programming the iRobot will affect the position and jerk profiles, these parameters should be included in such an investigation. This is not a trivial undertaking, but one which the results of this study can help to inform.

To gain a better representation of the footstrikes would require some modifications to the system and the results presented herein can help to inform this process. For both footstrikes the ground contact time was too long for the iRobot system (by an order of at least 2). This could be improved by increasing the speed of the iRobot movement; however, the maximum end-point velocity is limited to $2 \mathrm{~m} / \mathrm{s}$ [15]. During the ground contact phase of the human running trials the maximum foot velocity was approximately $4 \mathrm{~m} / \mathrm{s}$ and only exceeded $2 \mathrm{~m} / \mathrm{s}$ for the final 5-10 per cent of ground contact time, in agreement with the literature [20] and based on a running velocity typical of that used in human trials for wear testing of running shoes [24]. Given the relatively small increase in the maximum velocity required for the present application, then it may be expected that, by redesigning the end effector and redefining the end point, the mechanical advantage allows higher velocities to be achieved. For example, a rigid rod can be introduced between the terminal iRobot segment and prosthetic foot and the end point defined to be at the iRobot end of the rod, e.g. representing the shank with the end point located at the knee joint level. If the end point is programmed to move in an arc trajectory at $2 \mathrm{~m} / \mathrm{s}$, centred at an imaginary hip joint, then the radius of the arc at the foot level will be approximately twice that at the knee level and therefore the linear velocity will also be approximately twice that at the knee, i.e. up to approximately $4 \mathrm{~m} / \mathrm{s}$. Notably, the maximum end point velocity for the iRobot is very low compared with many velocities encountered in the sports domain. For example, golf and soccer have reported maximal ball velocities of approximately $80 \mathrm{~m} / \mathrm{s}$ and $34 \mathrm{~m} / \mathrm{s}$ respectively $[\mathbf{2 5}, \mathbf{2 6}]$. This requires club and leg velocities of approximately $60 \mathrm{~m} / \mathrm{s}$ and $28 \mathrm{~m} / \mathrm{s}$ respectively. Given that the iRobot has a state-of-the-art combined payload-end-point velocity capability, then for wider applications within the sports domain it will be necessary to investigate realistic end-point velocities that can safely be achieved through a mechanical advantage. An additional consideration regarding the use of a mechanical advantage relates to the increased driving load that could be placed on the iRobot compared with the current configuration. For human running, the higher velocities occur only during the final stages of ground contact where the ground reaction forces are far lower than their peak value. Therefore, it is expected that the velocities could be achieved with no change in the iRobot peak driving effort. Further sources of discrepancy in the ground contact time may have resulted from the effect of smoothing of the end-effector trajectory on the velocity profile and the nature of the ground contact phase. The low peak loading rates will have been the result of both the increased contact time and the increased trajectory smoothing, and improvements to these are the first stage to improving the loading rates. However, the magnitudes of the differences between the loading rates for the human and for the iRobot appear greater than those in the contact time and the smoothing alone; thus, to match force-based parameters may require further changes within the iRobot system such as the introduction of force feedback. This will increase the complexity of the system and therefore should be carried out after the more simple improvements are considered.

The MPJ angle also showed quite large discrepancies between human running and the iRobot system. Although the prosthetic foot showed the right trends, it was unable to flex as much as a human foot during ground contact, leading to a much straighter foot throughout both running techniques. Furthermore, the iRobot provided a horizontal braking force throughout the contact phase for both forefoot running and heelstrike running. This was achievable since, unlike human running, the iRobot is driven through the movement and does not need to propel itself forwards by pushing off the ground. This points to a requirement for an end-effector design that is better able to mimic the range of motion of the human MPJ. Specifically, the development of a new end effector will need to focus on matching the dynamic properties of the human heel pad at heelstrike, and 
the torque-angle properties of the MPJ during the active push-off phase. These observations suggest that matching the heel marker kinematics between the human and iRobot system may not have been the best reference point, i.e. the point on the shoe taken from the human running trials and used in the point-to-point programming of the iRobot.

These results indicate a potential disparity between artificial feet required for research studies such as that described herein and prosthetic feet designed for amputees. For research the exact requirements and hence foot design will depend on the specifics of the investigation; within the footwear industry this is likely to involve replicating the key kinematics and loading characteristics of the shod human foot during ground contact. In contrast, an amputee demands a prosthetic foot to improve or restore function, and to provide mobility that is safe, comfortable, and reliable rather than to mimic closely the dynamics of a human foot.

Although the footwear-testing capabilities of the iRobot system require further work in a number of identified areas, the fundamental potential to replicate characteristics of the human footstrike has been demonstrated. Indeed, the potential of the iRobot system as a useful research tool has been further explored through a pilot study conducted into the effect of wear on artificial surface hardness. Synchronous high-speed video and ground contact forces were captured from an experienced rugby player during sidestepping and running motions using the same equipment and set-up as described in section 2. This was repeated for four different surface designs and the human movements replicated on the iRobot. Surfaces were exposed to 2000 impacts of each movement (estimated to represent approximately 3 months' wear for a pitch used $48 \mathrm{~h}$ per week) and their properties measured at regular intervals (every 500 impacts). Surface hardness, as measured with a $2.25 \mathrm{kgf}$ Clegg hammer, increased significantly with increasing number of impacts for all surfaces and movements. This study supported the potential of the iRobot for applications such as studying the wear characteristics of artificial surfaces under realistic but controlled laboratory conditions.

\section{CONCLUSIONS}

Overall, this study has supported the potential of the iRobot system as a flexible and useful research and development tool within the sporting goods sector and in particular to enhance the one-dimensional mechanical tests currently utilized. The kinematic and contact force repeatability for the iRobot system was high in all parameters considered except the forefoot peak vGRF. Application of the iRobot system to replicate the ground contact phase of human running was achieved with limited success and also helped to identify a number of potential improvements that could be made to the system. These include a more realistic end-effector, 3D input kinematics, and the inclusion of force feedback in controlling the iRobot movement.

\section{ACKNOWLEDGEMENTS}

Sincere appreciation is extended to Blatchford and Sons for providing the Elite prosthetic foot and to the Engineering and Physical Sciences Research Council and UK Sport for providing the funding for this project.

\section{(C) Authors 2010}

\section{REFERENCES}

1 Cavanagh, P. R. Current approaches, problems, and future directions in shoe evaluation techniques. In Biomechanics, vol. IX-B, 1985, pp. 123-127 (Human Kinetics, Champaign, Illinois).

2 Odenwald, S. Test methods in the development of sports equipment. Engng Sport, 2006, 6, 302-306.

3 Cheung, J. T., Yu, J., Wong, D. W., and Zhang, M. Current methods in computer-aided engineering for footwear design. Footwear Sci., 2009, 1(1), 31-46.

4 Bates, B. T. Testing and evaluation of running shoes. In Biomechanics, vol. IX-B, 1985, pp. 128-132 (Human Kinetics, Champaign, Illinois).

5 Schempf, H. Roboleg: a robotic soccer-ball kicking leg. In Proceedings of the IEEE International Conference on Robotics and automation, 1995, pp. 1314-1319 (IEEE, New York).

6 Kotze, J. A tennis serve impact simulation machine. Engng Sport, 2002, 4, 477-484.

7 Harper, T. Robotic simulation of golf swings. $\mathrm{PhD}$ Thesis, Loughborough University, Loughborough, UK, 2007.

8 Ronkainen, J., Holmes, C., Harland, A., and Jones, R. A comparative study of ball launch measurement systems; soccer case study. Engng Sport, 2006, 7, 239-246.

9 Cavanagh, P. R., Morag, E., Boulton, A. J. M., Young, M. J., Deffner, K. T., and Pammer, S. E. The relationship of static foot structure to dynamic foot function. J. Biomech., 1997, 30(3), 243-250.

10 Monckton, S. P. and Chrystall, K. Design and development of an automated footwear testing system. In Proceedings of the IEEE International Conference on Robotics and automation, 2002, pp. 3684-3689 (IEEE, New York).

11 Lewis, G., Artoun, O., Griffin, K., Lim, J., Lio, X. P., Odegaard, J., Westbrook, R., and Williams, S. Forcecontrol for the automated footwear testing system. Can. Appl. Math. Q., 2005, 13(3), 249-264. 
12 Cook, S. D., Kester, M. A., and Brunet, M. E. Shock absorption characteristics of running shoes. Am. J. Sports Med., 1985, 13, 248-253.

13 Calder, C. A., Smith, C. E., and Ying, J. Measurement of shock-absorption characteristics of athletic shoes. Expl Techqs, 1985, 9(6), 21-24.

14 Roy, J. R. and Stefanyshyn, D. J. Shoe midsole longitudinal bending stiffness and running economy, joint energy, and EMG. Med. Sci. Sports Exercise, 2006, 38(3), 562-569.

15 Fanuc, Multi-purpose intelligent robot, Fanuc robot R-2000iB series, 2009, available from www.fanuc.co. jp/en/product/robot/lineup/r2000ib.html (access date 8 August 2009).

16 Newell, K. M. and Corcos, D. M. Variability and motor control, 1993 (Human Kinetics, Champagne, Illinois).

17 Winter, D. Kinematic and kinetic patterns in human gait variability and compensating effects. Hum. Movement Sci., 1984, 3, 51-76.

18 Giakas, G. and Baltzopoulos, V. Time and frequency domain analysis of ground reaction forces during walking: an investigation of variability and symmetry. Gait Posture, 1997, 5, 189-197.

19 White, S. C., Yack, J. H., Tucker, C. A., and Lin, H.-Y. Comparison of vertical ground reaction forces during overground and treadmill walking. Med. Sci. Sports Exercise, 1998, 30(10), 1537-1542.

20 de Wit, B., De Clerq, D., and Aerts, P. Biomechanical analysis of the stance phase during barefoot and shod running. J. Biomech., 2000, 33, 269-278.

21 Clarke, T. E., Frederick, E. C., and Hamill, C. L. The effects of shoe design parameters on rearfoot control in running. Med. Sci. Sports Exercise, 1983, 15(5), 376-381.
22 Perry, S. D. and Lafortune, M. A. Influences of inversion/eversion of the foot upon impact loading during locomotion. Clin. Biomech., 1995, 10(5), 253-257.

23 Cavanagh, P. R. and Lafortune, M. A. Ground reaction forces in distance running. J. Biomech., 1980, 13(5), 397-406.

24 Heinenfelder, J., Sterzing, T., and Milani, T. L. Biomechanical wear testing of running shoes. Footwear Sci., 2009, 1 (1), 16-17.

25 Naruo, T. and Mizota, T. Trajectory analysis and optimum trajectory of a golf ball. Engng Sport, 2004, 5, 432-439.

26 Neilson, P., Jones, R., Kerr, D., and Sumpter, C. An automated system for the measurement of soccer ball flight characteristics. Engng Sport, 2004, 5, 180-186.

\section{APPENDIX}

\section{Notation}

BW body weight

COP centre of pressure

$\mathrm{CV}$

hGRF

MPJ

MSD

SD

vGRF

$x$

$y$

$z$ coefficient of variation

horizontal ground reaction force metatarsophalangeal joint mean standard deviation standard deviation vertical ground reaction force medial-lateral axis anterior-posterior axis vertical axis 